\title{
A Selective History of the Stone-von Neumann Theorem
}

\author{
Jonathan Rosenberg
}

\begin{abstract}
The names of Stone and von Neumann are intertwined in what is now known as the Stone-von Neumann Theorem. We discuss the origins of this theorem, the contributions to it of Stone and von Neumann, the ways the theorem has been reformulated, and some of the varied mathematics that has grown out of it. At the end we discuss certain generalizations or analogues of the Stone-von Neumann Theorem which are still subjects of current research, such as a new $C^{*}$-algebra attached to the canonical commutation relations of quantum field theory, and supersymmetric versions of the theorem.
\end{abstract}

\section{Introduction}

As this volume is dedicated in honor of the hundredth birthdays of Stone and von Neumann, it seems totally appropriate to consider the history of the one theorem that bears both of their names. This history turns out to involve a surprisingly large swath of 20th century mathematics: from quantization to group representations, operator algebras, and even number theory. For other points of view on topics related to the Stone-von Neumann Theorem, I would recommend [24] and [59]. I want to thank Bob Doran and Dick Kadison for organizing a fascinating session in Baltimore and for originating the idea of this volume.

Some of the original sources analyzed here, especially in sections 1 and 2 , are in German, and some of the sources in section 4 are in French. To make this paper understandable to as wide an audience as possible, I have translated some of the relevant passages in footnotes. Any mistakes in the translations are clearly my responsibility.

\section{The early history of quantum mechanics, and origins of the problem}

I am not competent to explain in detail the early history of quantum mechanics, though there is no doubt that this is a fascinating chapter in the history of science. So this section will be very brief, and will concentrate just on the problem that motivated Stone and von Neumann. This problem concerns the "Heisenberg commutation relations." The name is a bit of a misnomer; the relations were in fact

2000 Mathematics Subject Classification. Primary 01A60; Secondary 22D10, 22D30, 22D25, 81R05, 81Q60, 81S05, 46L60.

This work was partially supported by NSF grant DMS-0103647. 
first formulated in their modern form not by Heisenberg but by Born and Jordan ${ }^{1}$ [4, equation (38)] and by Dirac [8, equation (11)] in the one-dimensional case, and in the "Dreimännerarbeit" ${ }^{2}$ by Born, Heisenberg, and Jordan [5, Chapter 2] and by Dirac in $[\mathbf{8}$, equation (12)], [9] in the multi-dimensional case. However it is true that they grew out of the original ground-breaking work of Heisenberg [20] (received by the journal July 29, 1925), though one would have to examine Heisenberg's paper very carefully to find anything remotely suggesting the commutation relations.

Nowadays we tend to think of quantum mechanics as a single subject, with the contributions of the various pioneers, such as Heisenberg and Schrödinger, blurred together. However, back in the 1920's, the mathematical foundations of the subject were still not worked out, and it was not clear if "matrix mechanics" (as developed by Heisenberg, Born, Dirac, and others) and "wave mechanics" (as developed primarily by Schrödinger) were equivalent. In a paper in 1926, Schrödinger alluded to this confusion and wrote [50, p. 735]: "Im folgenden soll nun der sehr intime innere Zusammenhang der Heisenbergschen Quantenmechanik und meiner Undulationsmechanik aufgedeckt werden. Vom formal mathematischen Standpunkt hat man ihn wohl als Indentität (der beiden Theorien) zu bezeichnen. Der Gedankengang des Beweises ist folgender." 3

Similarly, in Heisenberg's book [21], published in 1930 and based on lectures given in 1929, in the concluding section entitled "Der mathematische Apparat der Quantentheorie," ${ }^{4}$ Heisenberg writes (pp. 109-110): "Die Tatsache, daß Partikelbild und Wellenbild zwei verschiedene Erscheinungsformen ein- und derselben physikalischen Realität sind, bildet das zentrale Problem der Quantentheorie." ${ }^{5}$

The work of Stone and von Neumann grew out of the mathematical challenge implicit in these statements of Schrödinger and Heisenberg. However, before we get to Stone and von Neumann, it is necessary to mention the important work of Weyl, and especially the 1928 edition of his famous book Gruppentheorie und Quantenmechanik ${ }^{6}[\mathbf{6 5}]$, cited by Stone as motivation for his work.

\footnotetext{
${ }^{1}$ Heisenberg does give the usual formulation in a letter to Pauli [43, \#98, pp. 236-241], dated September 18, 1925, but he says there: "Die mir z. Z. sehr am Herzen liegende Quantenmechanik hat inzwischen, hauptsächlich durch Born und Jordan, entschiedene Fortschritte gemacht, die ich Ihnen, schon um mir selbst klar zu werden, was ich glauben soll, im Folgenden erzählen werde." Translation: "In the meantime the quantum mechanics which is currently very dear to my heart has made some crucial progress, largely through Born and Jordan ...." When Heisenberg writes down the commutation relation a few paragraphs later, he calls it "eine sehr gescheite Idee von Born," "a very clever idea of Born." The paper of Born and Jordan was received by the Zeitschrift für Physik on the 27th of September, only 9 days later, so it must have been basically complete by the date of Heisenberg's letter to Pauli, and Heisenberg already knew about it. The "Dreimännerarbeit" was received by the same journal on November 16, only about 2 months later.

2Translation: "three-man work." This nickname is an allusion to the fact that before World War II, collaborations involving more than two people were quite unusual in mathematics or theoretical physics.

${ }^{3}$ The italics are original. Translation: "In the following, only the very close inner connection between Heisenberg's quantum mechanics and my wave mechanics will be uncovered. From a formal mathematical point of view, one really has to prove the identity of the two theories. The train of thought of a proof follows."

4Translation: "The mathematical apparatus of quantum theory."

${ }^{5}$ Translation: "The fact that the particle picture and the wave picture are two different aspects of one and the same physical reality forms the central problem of quantum theory." The wave picture corresponds to Schrödinger's wave mechanics, the particle picture to matrix mechanics, which as Dirac pointed out, is founded on a formalism derived from Hamiltonian mechanics.

${ }^{6}$ Translation: Group Theory and Quantum Mechanics.
} 
Let us begin with the Heisenberg commutation relations, say for a free particle with one degree of freedom:

$$
P Q-Q P=-i \hbar .
$$

Here $P$ and $Q$ are supposed to be self-adjoint operators on a Hilbert space $\mathcal{H}$, representing momentum and position, respectively. ${ }^{7}$ (Stone replaces $-i$ by $i$; this clearly has no significance, as the laws of the universe should be invariant under $\operatorname{Gal}(\mathbb{C} / \mathbb{R})$, but we've retained the physicists' usual sign convention.)

The first observation to make is that (1) has no solutions if $\mathcal{H}$ is finite-dimensional and $\hbar \neq 0$, since the trace of any commutator must vanish, but $\operatorname{Tr}(-i \hbar)=$ $-i \hbar \operatorname{dim} \mathcal{H}$ does not. But (1) also has no solutions with either $P$ or $Q$ bounded. $^{8}(P$ and $Q$ play symmetrical roles, since interchanging them amounts to replacing $-i$ by $i$, i.e., to selecting a different square root of -1 , or to acting by $\operatorname{Gal}(\mathbb{C} / \mathbb{R})$.) A simple way to prove this is to introduce the Weyl integrated form of the relations, which first appears in $[\mathbf{6 5}, 1$ st ed., $\S 45$, or 2nd ed., Ch. IV, $\S 14]$.

Namely, we assume that $P$ and $Q$ can be exponentiated to one-parameter unitary groups $U_{\tau}=\exp (i \tau P)$ and $V_{\tau}=\exp (i \tau Q)$, respectively ( $\tau$ real). (This is possible by "Stone's Theorem" [57] if $P$ and $Q$ are self-adjoint.) Now (1) implies certain algebraic relations for $U_{\tau}$ and $V_{\tau}$. For example, we obtain formally

$$
\begin{aligned}
U_{\tau} Q U_{-\tau} & =\operatorname{Ad}(\exp (i \tau P)) Q \\
& =e^{\operatorname{ad}(i \tau P)}(Q)=Q+i \tau[P, Q]+\frac{(i \tau)^{2}}{2 !}[P,[P, Q]]+\cdots \\
& =Q+\tau \hbar \quad(\text { since }[P, Q]=-i \hbar \text { is a scalar }),
\end{aligned}
$$

so $Q$ is unitarily equivalent to $Q+\tau \hbar$ for any real $\tau$. (This formal calculation can be justified pointwise when applied to a vector in the range of a spectral projection of $Q$.) Thus if $\hbar \neq 0,(2)$ then proves the spectrum of $Q$ must consist of the whole real line, and in particular, $Q$ is unbounded.

Also, from (1) we immediately obtain that $U_{\tau} f(Q) U_{-\tau}=f(Q+\tau \hbar)$, for any real analytic function $f$ on the spectrum of $Q$, and in particular, we have

$$
\begin{aligned}
U_{\tau} V_{\sigma} U_{-\tau} & =U_{\tau} e^{i \sigma Q} U_{-\tau} \\
& =\exp (i \sigma(Q+\tau \hbar))=e^{i \sigma \tau \hbar} V_{\sigma},
\end{aligned}
$$

the multiplicative form of the commutation relations discovered by Weyl [65, 1st ed., $\S 45,(113)]$. Equation (3) is the starting point for the work of Stone and von Neumann. Weyl claims (in both editions of his book):

Das Postulat der Irreduzibilität bewirkt es, daß aus den Heisenbergschen Vertauschungsrelationen der besondere Ansatz mit Notwendigkeit folgt. ${ }^{9}$

Standards of proof are certainly not the same in physics and mathematics, and one can see this when comparing Weyl's book, which is full of heuristic arguments that can be made into proofs, even if they are not what mathematicians would call

\footnotetext{
${ }^{7}$ The idea of specifically requiring a Hilbert space, rather than an arbitrary complex vector space, seems to be due to von Neumann [61].

${ }^{8}$ This fact is implicit in the work of Weyl [65], though he never stated it formally. Proofs were later given by Wielandt [66] and Wintner [67].

${ }^{9}$ Translation: The assumption of irreducibility guarantees that from the Heisenberg commutation relations, the desired form [of the solution] necessarily follows.
} 
proofs, with the later paper of Stone, where it is asserted [57, p. 175] that "The significance of the last two theorems [the quote above and the earlier assumption that a self-adjoint operator can be exponentiated to a one-parameter unitary group] for the quantum mechanics has been pointed out by Weyl, who made no attempt to prove them." In the second edition of [65], published shortly after the work of Stone and von Neumann, Weyl adds a footnote:

Ein strenger Beweis dieses Satz im unendlich-dimensionalen Raum wurde angekündigt von M. H. Stone [57]; durchgeführt ist ein solcher Beweis auf anderer Grundlage, wie ich einer brieflichen Mitteilung entnehme, kürzlich von J. v. Neumann. ${ }^{10}$

\section{The work of Stone and von Neumann}

The beginnings of what we now call the Stone-von Neumann Theorem are in [57], which bears the date "communicated January 10, 1930." The section of this paper which is relevant for us consists only of one short paragraph (in which we've corrected one misprint), followed by the statement of a theorem:

A second question of group theory, to which we can apply the operational calculus, is raised by the Heisenberg permutation relations connecting the self-adjoint transformations $P_{k}, Q_{k}, k=$ $1, \ldots, n$. For convenience, we write these relations in the form

$$
\begin{aligned}
P_{k} Q_{l}-Q_{l} P_{k} & =i \delta_{k l} I, \quad P_{k} P_{l}-P_{l} P_{k}=0, \\
Q_{k} Q_{l}-Q_{l} Q_{k} & =0, \quad k, l=1, \ldots, n .
\end{aligned}
$$

In quantum mechanics, these transformations refer essentially to the coördinates and momenta of a dynamical system of $n$ degrees of freedom. The content of these permutation relations must be made precise by expressing them in terms of the one parameter groups of unitary transformations $U_{\tau}^{(k)}$ and $V_{\tau}^{(k)}$ generated by $i P_{k}$ and $i Q_{k}$, respectively. We have $U_{\sigma}^{(k)} V_{\tau}^{(k)}=$ $e^{-i \sigma \tau} V_{\tau}^{(k)} U_{\sigma}^{(k)}$, and $U_{\sigma}^{(k)} V_{\tau}^{(l)}=V_{\tau}^{(l)} U_{\sigma}^{(k)}$ when $k$ and $l$ are different, for $k, l=1, \ldots, n$; we also have $U_{\sigma}^{(k)} U_{\tau}^{(l)}=U_{\tau}^{(l)} U_{\sigma}^{(k)}$ and $V_{\sigma}^{(k)} V_{\tau}^{(l)}=V_{\tau}^{(l)} V_{\sigma}^{(k)}$ for $k, l=1, \ldots, n$. We prove the following theorem: ${ }^{11}$

THEOREM 2.1. If the family of transformations

$$
U_{\sigma_{1}}^{(1)} \cdots U_{\sigma_{n}}^{(n)} V_{\tau_{1}}^{(1)} \cdots V_{\tau_{n}}^{(n)}
$$

is irreducible in $\mathfrak{H}$, there there exists a one-to-one linear isometric correspondence or transformation which takes $\mathfrak{H}$ into $\mathfrak{H}_{n}$, the space of all complex-valued Lebesgue-measurable functions $f\left(x_{1}, \ldots, x_{n}\right),-\infty<x_{1}<+\infty, \ldots,-\infty<x_{n}<+\infty$, for which the integral

$$
\int_{-\infty}^{+\infty} \cdots \int_{-\infty}^{+\infty}\left|f\left(x_{1}, \ldots, x_{n}\right)\right|^{2} d x_{1} \ldots d x_{n}
$$

\footnotetext{
${ }^{10}$ Translation: A strict proof of this theorem in infinite dimensions was announced by M. H. Stone [57]; such a proof based on other principles has recently been carried out by J. v. Neumann, as I've learned from a letter. [Note Weyl's implicit distinction between "proof" and "strict proof."]

${ }^{11}$ I have numbered the theorem. In the original paper, it is unnumbered.
} 
exists, such that

$$
\begin{aligned}
S P_{k} S^{-1} f\left(x_{1}, \ldots, x_{n}\right) & =i \frac{\partial}{\partial x_{k}} f, \\
S Q_{k} S^{-1} f\left(x_{1}, \ldots, x_{n}\right) & =x_{k} f .
\end{aligned}
$$

Stone then proceeds to give only the barest hint of a proof, based on the operators

$$
T_{k}=Q_{k} P_{k} Q_{k}-P_{k}, \quad S_{k}=\left(Q_{k}-i\right) /\left(Q_{k}+i\right) .
$$

Stone says:

The principal difficulty lies in showing the transformations $T_{k}$ are self-adjoint. The determination of their spectra, under the hypothesis of irreducibility, can then be effected and leads easily to the construction of the desired transformation $S$ by means of a device previously employed by J. v. Neumann in a rather different connection. ${ }^{12}$

Von Neumann's contribution to the subject may be found in his paper [62], entitled "Die Eindeutigkeit der Schrödingerschen Operatoren." Unlike Stone's paper, it contains complete proofs. Because of the paper's significance, we reproduce here the first paragraph ${ }^{13}$ (by kind permission of the copyright holder, Springer-Verlag GmbH \& Co.) :

\section{Die sogenannte Vertauschungsrelation}

$$
P Q-Q P=\frac{h}{2 \pi i} 1
$$

ist in der neuen Quantentheorie von fundamentaler Bedeutung, sie ist es, die den "Koordinaten-Operator" $R$ und den "Impuls-Operator" $P$ im wesentlichen definiert $\left.{ }^{1}\right)$. Mathematisch gesprochen, liegt darin die folgende Annahme: Seien $P, Q$ zwei Hermitesche Funktionaloperatoren des Hilbertschen Raumes, dann werden sie durch die Vertauschungsrelation bis auf eine Drehung des Hilbertschen Raumes, d. i. eine unitäre Transformation $U$,

\footnotetext{
${ }^{12}$ Stone here has a footnote to von Neumann's work on the Cayley transform of an unbounded self-adjoint operator.

${ }^{13}$ Translation: The so-called commutation relation (1) is of fundamental importance in the new quantum theory [5] [8]; here $R$ [an obvious typo-he means $Q$ ] is the coordinate operator and $P$ is the momentum operator. Mathematically speaking, the following assumptions are implicit: $P$ and $Q$ are hermitian operators on a Hilbert space, which up to a rotation of the Hilbert space, i.e., up to conjugation by a unitary transformation, are uniquely determined. It's required that we impose an extra condition: that $P$ and $Q$ should form an irreducible system. If we now, as in Schrödinger's particularly convenient formulation of the quantum theory, interpret the Hilbert space as a function space - the simplest being the space of all complex functions $f(q)$, $-\infty<q<\infty$, with $\int|f(q)|^{2} d q<\infty$ - then we have following Schrödinger [50] a particularly simple solution of the commutation relation, namely
}

$$
Q: f(q) \mapsto q f(q), \quad P: f(q) \mapsto-i \hbar \frac{d}{d q} f(q)
$$


eindeutig festgelegt ${ }^{2}$ ). Es liegt im Wesen der Sache, daß noch der Zusatz gemacht werden muß: vorausgesetzt, daß $P, Q$ ein irreduzibles System bilden (vgl. weiter unten Anm. ${ }^{6}$ )). Wird nun, wie es sich durch die Schrödingersche Fassung der Quantentheorie als besonders günstig erwies, der Hilbertsche Raum als Funktionenraum interpretiert - der Einfachheit halber etwa als Raum aller komplexen Funktionen $f(q)(-\infty<q<+\infty)$ mit endlichem $\int_{-\infty}^{+\infty}|f(q)|^{2} d q-$, so gibt es nach Schrödinger ein besonders einfaches Lösungssystem der Vertauschungsrelation

$$
\left.Q: f(q) \rightarrow q f(q), \quad P: \quad f(q) \rightarrow \frac{h}{2 \pi i} \frac{d}{d q} f(q)^{3}\right) .
$$

Von Neumann then goes on to ask:

Sind nun dies die im wesentlichen einzigen (irreduziblen) Lösungen der Vertauschungsrelation? ${ }^{14}$

Es bliebe daher zu zeigen, daß die einzigen irreduziblen Lösungen der Weylschen Gleichungen die Schrödingerschen sind. Beweisansätze heifür gab Stone an, jedoch ist bisher ein Beweis auf dieser Grundlage, wie mir Herr Stone freundlichst mitteilte, nich erbracht worden.

Im folgenden soll der genannte Eindeutigkeitsatz bewiesen werden. ${ }^{15}$

Now let's analyze in modern terms what Stone and von Neumann did. We start with the Canonical Commutation Relations:

$$
\left[P_{j}, P_{k}\right]=0, \quad\left[Q_{j}, Q_{k}\right]=0, \quad\left[P_{j}, Q_{k}\right]=-i \delta_{j k} \hbar,
$$

$P_{j}, Q_{k}$ self-adjoint, $1 \leq j, k \leq n$. The unbounded operator problems are substantial, since the $P$ 's and $Q$ 's are not everywhere defined, while the right-hand side of each equation is a scalar. (In fact there is a substantial literature on solutions of the commutation relations that do not necessarily integrate to unitary groups, and for these the Stone-von Neumann Theorem breaks down completely, but this is a subject for a different paper.) We bypass these difficulties by going to the Weyl integrated form. In other words, assume we have unitary representations $U, V$ of $\mathbb{R}^{n}$, obtained by "integrating" the $P$ 's and $Q$ 's, respectively. Thus the result can be phrased as follows:

Theorem 2.2. Consider pairs $(U, V)$ of unitary representations of $\mathbb{R}^{n}$ on a Hilbert space $\mathcal{H}$, satisfying the commutation rule

$$
U(x) V(y)=\exp (i \omega(x, y)) V(y) U(x)
$$

\footnotetext{
${ }^{14}$ Translation: Are these now the essentially unique (irreducible) solution of the commutation relation?

${ }^{15}$ Translation: It remains therefore to show that Schrödinger's solution is the unique irreducible solution of the Weyl equations. Stone has previously given hints of a proof, but a proof based on these principles, as Mr. Stone has in a friendly way communicated to me, has not been completed. In what follows, the desired uniqueness theorem will be proven.
} 
$\omega: \mathbb{R}^{n} \times \mathbb{R}^{n} \rightarrow \mathbb{R}$ bilinear and non-degenerate. Such pairs are all equivalent to multiples of the standard Schrödinger representation on $L^{2}\left(\mathbb{R}^{n}\right)$.

Note that we can combine $U$ and $V$ into a projective unitary representation of $\mathbb{R}^{2 n}$, or equivalently into a unitary representation of what is usually called the Heisenberg group ${ }^{16}$

$$
H=\left\{\left(\begin{array}{ccccc}
1 & x_{1} & \cdots & x_{n} & z \\
0 & 1 & \cdots & 0 & y_{n} \\
0 & 0 & \ddots & 0 & \vdots \\
0 & 0 & \cdots & 1 & y_{1} \\
0 & 0 & \cdots & 0 & 1
\end{array}\right): x_{j}, y_{k}, z \in \mathbb{R}\right\}
$$

with specified central character $z \mapsto e^{i \hbar z}$.

Since it will be relevant later, let's briefly sketch a proof of Theorem 2.2 along the lines of von Neumann's paper. To begin with, note that since any nondegenerate bilinear skew-symmetric form on $\mathbb{R}^{2 n}$ is equivalent to the standard form given by $\left(\begin{array}{cc}0_{n} & 1_{n} \\ -1_{n} & 0_{n}\end{array}\right)$, this formulation is really equivalent to Stone's in Theorem 2.1. So without loss of generality, we may assume the relations reduce to saying that

$$
U(x) V(y)=e^{i\langle x, y\rangle} V(y) U(x),
$$

with $\langle$,$\rangle the usual Euclidean inner product on \mathbb{R}^{n}$.

The key observation is then that given any representation of the relation (5) on a Hilbert space $\mathcal{H}$, one obtains a large number of self-adjoint projections on $\mathcal{H}$ of the form

$$
P_{\varphi}=\iint U(x) V(y) \varphi(x, y) d x d y
$$

$\varphi \in \mathcal{S}\left(\mathbb{R}^{2 n}\right)$ (the Schwartz space of rapidly decreasing functions). Indeed, if $P_{\varphi}$ is given by (6), then the required relations $P_{\varphi}=P_{\varphi}^{*}=P_{\varphi}^{2}$ are satisfied if

$$
\varphi(-x,-y)=e^{-i\langle x, y\rangle} \overline{\varphi(x, y)}
$$

and

$$
\iint \varphi(x, y) \varphi(u-x, v-y) e^{i\langle x-u, y\rangle} d x d y=\varphi(u, v) .
$$

Furthermore, von Neumann proves (by Fourier analysis) that the operator $P_{\varphi}$ can vanish only if $\varphi \equiv 0$. As noticed by von Neumann, if

$$
\varphi(x, y)=\frac{1}{(2 \pi)^{n}} e^{-i\langle x, y\rangle / 2} e^{-\left(|x|^{2}+|y|^{2}\right) / 4},
$$

then the conditions for $P_{\varphi}=P_{\varphi}^{*}=P_{\varphi}^{2}$ are satisfied. Furthermore, one finds with this choice that $P_{\varphi} U(x) P_{\varphi}$ and $P_{\varphi} V(y) P_{\varphi}$ agree with $P_{\varphi}$ up to scalar factors depending of course on $x$ and $y$. Thus if the representation of the $U(x)$ 's and $V(y)$ 's on $\mathcal{H}$ is irreducible, $P_{\varphi}$ must be a rank-one projection. ${ }^{17}$ Theorem 2.2 follows, since given two irreducible representations of (5) on $\mathcal{H}$ and $\mathcal{H}^{\prime}$, respectively, the map

\footnotetext{
${ }^{16}$ As should be clear by now, this is not Heisenberg's invention, so the name is a bit of a misnomer. The central extension of $\mathbb{R}^{2 n}$ by the circle group $\mathbb{T}$, a non-simply connected Lie group with $H$ as its universal cover, was really discovered by Weyl.

${ }^{17}$ If $\operatorname{rk} P_{\varphi}>1$, then we can write $P_{\varphi}$ as the sum of two proper subprojections, and one can check that these will generate proper invariant subspaces of $\mathcal{H}$, contradicting irreducibility.
} 
sending a unit vector in the range of $P_{\varphi}$ on $\mathcal{H}$ to a unit vector in the range of the corresponding operator $P_{\varphi}^{\prime}$ on $\mathcal{H}^{\prime}$ will extend uniquely to a unitary intertwining operator. Variations on this argument will play a crucial role in section 5 below.

At this point we can return to the original questions of Schrödinger and Heisenberg, concerning the equivalence of matrix and wave mechanics, or of the particle and wave pictures. The Stone-von Neumann Theorem answers these questions affirmatively in the case of a single free particle, in the sense that all irreducible representations of the fundamental relations (4), whether on configuration space (the "particle picture"), momentum space (the "wave picture"), or some other abstract Hilbert space ("matrix mechanics"), are unitarily equivalent to one another.

\section{Mackey's version}

The next major step in the history of the Stone-von Neumann Theorem was taken by George Mackey in his ground-breaking paper [36]. Incidentally, to my knowledge, the name "Stone-von Neumann Theorem" can be traced back to the title of this paper of Mackey.

TheOREM 3.1 (Mackey $[\mathbf{3 6}]$ ). Let $G$ be a locally compact group. Then any covariant pair of representations of $G$ and $C_{0}(G)$ on a Hilbert space $\mathcal{H}$ is a multiple of the standard representation on $L^{2}(G)$. (A covariant pair is $(\pi, \alpha)$, where $\pi$ is a unitary representation of $G$ on $\mathcal{H}, \alpha$ is a *-representation of $C_{0}(G)$ on $\mathcal{H}$, and $\left.\pi(g) \alpha(f) \pi(g)^{*}=\alpha(g \cdot f), g \cdot f(h)=f\left(g^{-1} h\right).\right)$

Mackey was aware that the Fourier transform makes it possible to identify unitary representations of a locally compact abelian group with representations of the algebra of functions on the Pontrjagin dual. Since $\mathbb{R}^{n}$ is self-dual, we can use this principle to convert the representation $V$ of $\mathbb{R}^{n}$ in Theorem 2.2 to a representation $\alpha$ of $C_{0}\left(\mathbb{R}^{n}\right)$. The situation of Stone and von Neumann is thus the case of $G=\mathbb{R}^{n}$. In general, for $G$ abelian, a covariant pair as in Theorem 3.1 is the same as a pair $(\pi, \sigma)$ with $\pi$ a representation of $G, \sigma$ a representation of $\widehat{G}$, and

$$
\pi(g) \sigma(\widehat{g}) \pi(g)^{*}=\langle g, \widehat{g}\rangle \sigma(\widehat{g}),
$$

which one recognizes as an obvious generalization of the commutation relation (5).

Theorem 3.1, often called the Stone-von Neumann-Mackey Theorem, was the first step in a long series of papers by Mackey (e.g., [37], [38], [39]) on induced representations. Mackey's Imprimitivity Theorem, the foundation of this work, can be stated in way that immediately generalizes Theorem 3.1:

ThEOREM 3.2 (Mackey [37]). Let $G$ be a locally compact group, $H$ a closed subgroup. Then any covariant pair $(\pi, \alpha)$ of representations $\pi$ of $G$ and $\alpha$ of $C_{0}(G / H)$ on a Hilbert space $\mathcal{H}$ is induced from a unitary representation $\sigma$ of $H$ on a Hilbert space $\mathcal{H}_{\sigma}$. In other words, we may identify $\mathcal{H}$ with the Hilbert space of measurable functions $f: G \rightarrow \mathcal{H}_{\sigma}$ such that

$$
f(g h)=\sigma(h)^{-1} f(g), \quad \int_{G / H}|f(g H)|^{2} d \dot{g}<\infty,
$$

and $g$ acts on this space by left translation: $\pi(g) f(x)=f\left(g^{-1} x\right)$. (For simplicity, we're tacitly assuming here that $G / H$ has a $G$-invariant measure $d \dot{g}$. The squareintegrability condition then makes sense because $|f(g)|^{2}$ is constant on cosets of $H$, even though $f$ itself is not, because of the covariance condition and unitarity of $\sigma$. 
If $G / H$ does not have a $G$-invariant measure, the formulas are similar, but one needs to use a quasi-invariant measure and insert various modular functions.)

Note that Theorem 3.1 is indeed a special case of Theorem 3.2, since if $H=\{1\}$, any representation $\sigma$ of $H$ is a multiple of the trivial one-dimensional representation, and thus any representation of $G$ induced from $H$ is a multiple of the standard representation of $G$ on $L^{2}(G)$.

The original approach of Mackey to Theorems 3.1 and 3.2 depended on a fair amount of measure theory, and required $G$ to be second countable (or "separable"). Subsequent simplifications in the proof of the Imprimitivity Theorem (see, e.g., [3] and $[\mathbf{4 2}]$ ) have made it possible to do away with the measure theory and with the separability hypothesis.

\section{The Shale-Weil representation}

Let $\mathbb{F}$ be a self-dual locally compact field, e.g., $\mathbb{F}_{q}, \mathbb{R}, \mathbb{C}$, or $\mathbb{Q}_{p}$. Self-duality means that if we regard $\mathbb{F}$ as a locally compact abelian group under addition and fix a non-trivial character $\chi: \mathbb{F} \rightarrow \mathbb{T}$, then $\chi$ enables us to identify the Pontrjagin dual $\widehat{\mathbb{F}}$ with $\mathbb{F}$ itself, in that every continuous character of $\mathbb{F}$ is of the form $x \mapsto \chi(x y)$, for some $y \in \mathbb{F}$. Slightly more accurately, if $V$ is a finite-dimensional vector space over $\mathbb{F}$, then once $\chi$ is fixed, we have a natural identification of $\widehat{V}$ with the vector space dual $V^{*}$ of $V$, in that every character of $V$ can be written uniquely as $\chi \circ \lambda$, $\lambda \in V^{*}$. Now let $(V, B)$ be a symplectic vector space of dimension $2 n$ over $\mathbb{F}$. The symplectic form $B$ identifies $V^{*}$ with $V$. So by Mackey's generalization of the Stone-von Neumann Theorem, ${ }^{18}$ there is a unique irreducible representation $\pi$ of the commutation relation

$$
\pi(v) \pi(w)=\chi(B(v, w)) \pi(w) \pi(v), \quad v, w \in V .
$$

(We are applying the theorem to $V$, regarded as an additive group.) There are of course many ways to realize this irreducible representation, but the simplest is the Schrödinger model, which goes back to the original ideas of the founders of quantum mechanics. Namely, we fix a polarization of $(V, B)$, that is, a decomposition $V=$ $X \oplus Y$ with $X$ and $Y$ both isotropic for $B$ (and thus, by dimension-counting, maximal isotropic). Then it suffices to find a covariant pair of representations of $X$ and of $Y$ as in Theorem 2.2, and we can realize the representation on $L^{2}(X)$ (which we can think of as "functions on configuration space"), with $X$ acting by translation and with $Y$ acting by multiplication by characters.

Since $\operatorname{Sp}(V, B)$ preserves the relation (8), Schur's Lemma implies there is a projective representation $\omega$ of $\operatorname{Sp}(V)$ on the Hilbert space of the Schrödinger representation, given by

$$
\omega(g) \pi(v) \omega(g)^{*}=\pi(g \cdot v) .
$$

(Equation (9) says $\omega(g)$ is an intertwining operator between two irreducible representations, and thus by Schur's Lemma it is uniquely determined up to a constant.)

Theorem 4.1 ([53, Theorem 5.1]; Weil [64]). The representation $\omega$ lifts to a true representation of a double cover of $\operatorname{Sp}(V)$.

${ }^{18}$ The original formulation of Stone and von Neumann applies precisely to the case $\mathbb{F}=\mathbb{R}, V$ the phase space (the direct sum of configuration space and momentum space) of a free particle. 
In fact, the work of Shale and Weil is more precise, and leads to explicit formulas for the cocycle defining this double cover. (Very readable "modernized" accounts are in $[\mathbf{3 4}$, Part I] and in [47].) For a few fields, such as $\mathbb{C}$ or finite fields of odd characteristic (see $[\mathbf{2 2}]),{ }^{19}$ the cocycle is a coboundary and $\omega$ can be regarded as a true representation of $\operatorname{Sp}(V)$, but this is more the exception than the rule.

As pointed out by Weil [64], the same theory also goes through not just locally but over the adèles $\mathbb{A}_{K}, K$ a number field. $\left(\mathbb{A}_{K}\right.$ is the restricted direct product $\prod_{v}^{\prime} K_{v}$ of all completions $K_{v}$ of $K$, both non-archimedean (finite extensions of $\mathbb{Q}_{p}$ for primes $p$ ) and archimedean (copies of $\mathbb{R}$ and $\mathbb{C}$ coming from the real and complex embeddings of $K)$. $K$ embeds in $\mathbb{A}_{K}$ as a discrete cocompact subgroup.) Let $V$ be a symplectic vector space over $K$ and let $V\left(\mathbb{A}_{K}\right)=\mathbb{A}_{K} \otimes_{K} V$. Then fixing a non-trivial additive character $\chi$ of $\mathbb{A}_{K}$, trivial on $K$, defines a Weil representation $\omega$ of a double cover of $\operatorname{Sp}\left(V\left(\mathbb{A}_{K}\right)\right)$. Furthermore, the inclusion $\operatorname{Sp}(V) \hookrightarrow \operatorname{Sp}\left(V\left(\mathbb{A}_{K}\right)\right)$ has a unique lifting into this double cover, so $\omega$ restricts to a (true) representation of $\operatorname{Sp}(V)$.

This Shale-Weil representation of the double cover of the symplectic group, also called (especially by Roger Howe) the oscillator representation, plays a fundamental role in representation theory and number theory. In fact, most of the correspondences in the theory of automorphic forms are derived from special cases of the theta correspondence based on this representation. This is not the place to go into details on this vast subject, so we will just give a few hints of what is involved and let the reader look elsewhere (e.g., in $[\mathbf{2 3}],[\mathbf{3 4}],[\mathbf{2 6}],[\mathbf{4 1}]$, and $[\mathbf{4 5}]$ ) for more information.

The theory of the theta correspondence is based on the notion of a reductive dual pair, introduced by Howe in [23]. This is a pair $(G, H)$ of reductive subgroups of the symplectic group $\operatorname{Sp}(V)$ which are each other's commutants inside the symplectic group. (Just as an aside, this was motivated in part by von Neumann's Double Commutant Theorem for operator algebras.) For example, if $V=V_{1} \otimes V_{2}$ with $B_{1}$ a non-degenerate symmetric bilinear form on $V_{1}, B_{2}$ a symplectic form on $V_{2}$, and $B=B_{1} \otimes B_{2}$, then we can take $G=O\left(V_{1}, B_{1}\right), H=\operatorname{Sp}\left(V_{2}, B_{2}\right)$. The restriction of the oscillator representation $\omega$ to [the double cover of] $G \times H$ decomposes, and the decomposition gives a correspondence between representations [possibly living on a double cover] of $G$ and those of $H$. (Roughly speaking, a representation $\pi$ of $G$ corresponds to a representation $\sigma$ of $H$ if $\pi \times \sigma$ occurs in this decomposition.) If one works in the correct categories of representations, then this correspondence, while only partially defined, is known (if one leaves aside a few special cases still being investigated) to be injective $([\mathbf{2 6}],[\mathbf{4 1}],[\mathbf{6 3}])$.

The name "theta correspondence" comes from a relationship between automorphic forms, associated to this correspondence of representations. This relationship is connected to the theory of theta functions, hence the name. In this context it is worth recalling the opening sentences of Weil's paper [64]:

À force d'habitude, le fait que les séries thêta définissent des fonctions modulaires a presque cessé de nous étonner. Mais l'apparition du groupe symplectique comme un deus ex machina

\footnotetext{
${ }^{19}$ One can even prove something about $\mathbb{F}_{2^{r}}$ if one excludes a small number of cases.
} 
dans les célèbres travaux de Siegel sur les formes quadratiques n'a rien perdu encore de son caractère mystérieux. ${ }^{20}$

We will content ourselves here with mentioning just one example. The standard classical theta function (that arises, for example, in the theory of the heat equation and in elliptic function theory) is

$$
\theta(z)=\sum_{n=-\infty}^{\infty} e^{\pi i n^{2} z}
$$

This series converges uniformly on compacta to an analytic function on the upper half-plane $\mathfrak{H}$, is clearly periodic of period 2 , and, by an application of the Poisson summation formula, satisfies the functional equation

$$
\theta\left(-\frac{1}{z}\right)=\sqrt{-i z} \theta(z)
$$

with the choice of the branch of the square root with $\sqrt{-i z}>0$ for $z$ on the positive imaginary axis. From this, the periodicity, and a little more work, one can prove [34, Theorem 2.4.13] that $\theta$ obeys a transformation law of the form

$$
\begin{aligned}
\theta\left(\frac{a z+b}{c z+d}\right)=\lambda & \left(\begin{array}{ll}
a & b \\
c & d
\end{array}\right)(c z+d)^{1 / 2} \theta(z), \\
& \left(\begin{array}{ll}
a & b \\
c & d
\end{array}\right) \in S L(2, \mathbb{Z}), a c \equiv 0 \quad \bmod 2, b d \equiv 0 \quad \bmod 2,
\end{aligned}
$$

with $\lambda$ a certain character taking values in the 8th roots of unity. The function $\theta$ is also important in number theory since

$$
\theta(z)^{k}=\sum_{n_{1}=-\infty}^{\infty} \cdots \sum_{n_{k}=-\infty}^{\infty} e^{\pi i\left(n_{1}^{2}+\cdots+n_{k}^{2}\right) z}=\sum_{n=0}^{\infty} a_{n} e^{\pi i n z},
$$

with $a_{n}$ the number of ways of representing the non-negative integer $n$ as a sum of $k$ squares.

The connection of the function $\theta$ with the oscillator representation is related to the following important fact:

Theorem 4.2 (Howe, [23, Theorem 4.1]). Let $K$ be a number field, $(V, B)$ a symplectic vector space over $K$. Then $(V, B)$ defines a Heisenberg group

$$
\begin{array}{r}
H=H(V, B)=V \oplus K \text { (as a set), with group operation } \\
(v, s) \cdot\left(v^{\prime}, s^{\prime}\right)=\left(v+v^{\prime}, s+s^{\prime}+B\left(v, v^{\prime}\right) / 2\right) .
\end{array}
$$

Fix a non-trivial additive character $\chi$ of $\mathbb{A}_{K}$ trivial on $K$ and let $\rho$ be the associated Stone-von Neumann representation of $H\left(\mathbb{A}_{K}\right)$ with central character given by $\chi$. Let $\omega$ be the associated oscillator representation of the double cover of $\operatorname{Sp}\left(V\left(\mathbb{A}_{K}\right)\right)$. Let $\mathcal{S}_{\rho}$ be the space of $C^{\infty}$ vectors for $\rho$. (This is a restricted tensor product of Schwartz spaces.) Then $\mathcal{S}_{\rho}$ is also the space $\mathcal{S}_{\omega}$ of $C^{\infty}$ vectors for $\omega$, and there is a distribution (i.e., continuous linear functional) $\Theta$ on $\mathcal{S}_{\rho}$, unique up to scalar multiples, which is invariant under $\rho(H)$ and under $\omega(\operatorname{Sp}(V(K)))$.

\footnotetext{
${ }^{20}$ Translation: Out of force of habit, the fact that theta series define modular functions has almost ceased to surprise us. But the appearance of the symplectic group as a deus ex machina in the celebrated work of [C. L.] Siegel on quadratic forms hasn't at all lost its mysterious character.
} 
Now let's specialize to the case $K=\mathbb{Q}, \operatorname{dim} V=2, B$ given by $\left(\begin{array}{cc}0 & 1 \\ -1 & 0\end{array}\right)$. Then the symplectic group can be identified with $S L(2)$. At the archimedean place $v=\infty, \mathcal{S}_{\rho}$ becomes $\mathcal{S}(\mathbb{R})$, with $\Theta$ giving a tempered distribution that is supposed to be invariant under translation by $\mathbb{Z}$ and multiplication by $e^{2 \pi i t}$ (since these generate $\rho\left(H_{\mathbb{Z}}\right)$, assuming $\chi$ is suitably normalized). Since $e^{2 \pi i t} \neq 1$ unless $t \in \mathbb{Z}, \Theta$ must be supported on the integer lattice, and thus it is obvious that $\Theta$ must be given (up to a scalar multiple) by the formula

$$
\Theta(f)=\sum_{n \in \mathbb{Z}} f(n)
$$

But now let $\xi(t)=e^{-\pi t^{2}}$, so that $\xi$ certainly lies in $\mathcal{S}(\mathbb{R})$. Consider $\varphi(g)=$ $\Theta(\omega(g) \xi)$, for $g \in \widetilde{S L}(2, \mathbb{R})$, the double cover of $S L(2, \mathbb{R})$. If $g_{t}$ is the usual lift of the one-parameter multiplicative group $\left(\begin{array}{cc}t & 0 \\ 0 & t^{-1}\end{array}\right), t>0$, in $S L(2, \mathbb{R})$, then we compute that

$$
\begin{aligned}
\varphi\left(g_{t}\right) & =\Theta\left(\omega\left(g_{t}\right) \xi\right) \\
& =\sum_{n \in \mathbb{Z}} f_{t}(n) \quad\left(f_{t}(s)=\omega\left(g_{t}\right) \xi(s)=\sqrt{t} e^{-\pi(t s)^{2}}\right) \\
& =\sqrt{t} \sum_{n \in \mathbb{Z}} e^{-\pi n^{2} t^{2}}=\sqrt{t} \theta\left(i t^{2}\right),
\end{aligned}
$$

which explains the connection between $\Theta$ and classical theta series.

As far as the "correspondence" aspect is concerned, the main idea is that if we have a reductive dual pair $(G, H)$ in $\operatorname{Sp}(V)$, then we can use the distribution $\Theta$ to "lift" automorphic forms from $G$ to $H$ or vice versa. More precisely, suppose $\xi$ lies in the Schwartz space on which the oscillator representation $\omega$ acts, and $f$ is an automorphic form for $G$ (meaning that $f$ is a smooth function on $\widetilde{G}(K) \backslash \widetilde{G}\left(\mathbb{A}_{K}\right)$ satisfying a suitable decay condition and generating an irreducible representation of $\widetilde{G}\left(\mathbb{A}_{K}\right)$ under right translation), then the integral

$$
\theta_{\xi}(f)(h)=\int_{\widetilde{G}(K) \backslash \widetilde{G}\left(\mathbb{A}_{K}\right)} \Theta(\omega(g h) \xi) f(g) d \dot{g},
$$

when it converges, gives a well-behaved function on $\widetilde{H}(K) \backslash \widetilde{H}\left(\mathbb{A}_{K}\right)$, that is, an automorphic form on $H$. (We are using the fact that $\Theta$ is invariant under $\widetilde{\operatorname{Sp}}(V(K)$ ), and thus under $\widetilde{G}(K) \times \widetilde{H}(K)$, by Theorem 4.2.) This can be used to "lift" automorphic forms from $G$ to $H$ (or vice versa). This kind of lifting turns out to be closely related to the local correspondence (between representations of $\widetilde{G}_{v}$ and representations of $\widetilde{H}_{v}$ ) discussed earlier.

\section{Rieffel's work and the notion of Morita equivalence}

The "modern" approach to the Stone-von Neumann Theorem, which is somewhat more algebraic, is due to Rieffel [48]. The key observation of Rieffel is that the theorem is really about an equivalence of categories of representations, or in the language of ring theory, a Morita equivalence. For example, the rings $\mathbb{C}$ and $M_{n}(\mathbb{C})$ are Morita equivalent; each of these has the property that every representation is a multiple of the unique irreducible representation. In a similar way, the category of (Weyl integrated forms) of representations of the commutation relations 
is equivalent to the category of representations of $\mathbb{C}$. Rieffel, following von Neumann's method of proof using the projections $P_{\varphi}$ of (6), showed this directly, and concluded that on any Hilbert space representation of the Heisenberg commutation relations, there is a natural action of $\lim _{\longrightarrow} M_{n}(\mathbb{C})$, or of its completion, the compact operators $\mathcal{K}$.

To explain this in a little more detail, the Morita theorems (see for example $[\mathbf{2 7}, \S 3.12]$ or $[\mathbf{3 3}, \S 18])$ say that two rings $A$ and $B$ have equivalent categories of (left) modules if and only if there are bimodules $X$ (for $A$ acting on the left, $B$ acting on the right) and $Y$ (for $B$ acting on the left, $A$ acting on the right) such that the equivalences in the two directions are implemented by the functors $M \mapsto Y \otimes_{A} M$ ( $M$ a left $A$-module) and $N \mapsto X \otimes_{B} N$ ( $N$ a left $B$-module), with $X \otimes_{B} Y \cong A$ (as an $A$ - $A$ bimodule) and $Y \otimes_{A} X \cong B$ (as a $B$ - $B$ bimodule). For example, when $A=\mathbb{C}$ and $B=M_{n}(\mathbb{C})$, one can take $X=\mathbb{C}^{n}$ (viewed as row vectors, $B$ acting by right matrix multiplication) and $Y=\mathbb{C}^{n}$ (viewed as column vectors). Rieffel adapted the same machinery, but with modifications appropriate for $*$-algebras and representations on inner product spaces. This modification was originally called strong Morita equivalence, but we will drop the adjective "strong" since this is the only kind of Morita equivalence we will be interested in for $C^{*}$ algebras.

The simplest case of a Morita equivalence (both in the algebraic setting and in Rieffel's theory), which in fact is almost ${ }^{21}$ the most general, is that of a full corner. ${ }^{21}$ We say $B$ is a full corner of $A$ if there is a projection $p$ in the multiplier algebra $^{22} M(A)$ of $A$ such that $B=p A p$ and $A=A p A$. (In the purely algebraic theory, we usually require $A$ and $B$ to be unital, $p \in A$ an idempotent. In the $C^{*}$-context, the algebras can be non-unital and $p$ is required to be self-adjoint.) The equivalence bimodule ${ }_{A} X_{B}$ is then $A p$. This situation clearly applies to the context of von Neumann's proof: $A$ is the $C^{*}$-algebra generated by all operators $\iint U(x) V(y) \psi(x, y) d x d y, p$ is the specific idempotent $P_{\varphi}$ constructed by von Neumann, and $B$ is one-dimensional, hence isomorphic to $\mathbb{C}$. Thus we obtain the desired Morita equivalence. We also see that $A \cong \mathcal{K}$, the algebra of compact operators on an infinite-dimensional separable Hilbert space.

Rieffel [49] went on to explain the Stone-von Neumann-Mackey Theorem, as well as the Mackey Imprimitivity Theorem, in similar terms. Covariant pairs of representations of $G$ and of $C_{0}(G / H)$, or in Mackey's language, systems of imprimitivity based on $G / H$, can be identified with representations of a crossed product algebra $C_{0}(G / H) \rtimes G$ which is Morita equivalent (now in the $C^{*}$-algebra sense) to the group $C^{*}$-algebra $C^{*}(H)$. The associated correspondence of representations matches induced representations Ind $\sigma$ of $G$ (which come with systems of imprimitivity) with the inducing representations $\sigma$ of $H$.

Morita equivalence for $C^{*}$-algebras has proved to be an extremely fruitful notion, as will be obvious from looking at the other papers in this volume.

\footnotetext{
${ }^{21}$ The meaning of "almost the most general" (in the $C^{*}$ context) is that if $A$ and $B$ are $C^{*}$-algebras, then they are Morita equivalent if and only if they can be simultaneously embedded as full corners in another $C^{*}$-algebra [6, Theorem 1.1].

${ }^{22}$ The multiplier algebra $M(A)$ is the largest $\left(C^{*}\right.$-)algebra in which $A$ embeds as an essential ideal. Of course, if $A$ is unital, then $M(A)=A$.
} 


\section{Non-perfect pairings and the work of Pukánszky and Baggett-Kleppner}

Recall that when $G$ is locally compact abelian, the Stone-von Neumann-Mackey Theorem deals with covariant pairs of representations of $G$ and $\widehat{G}$ satisfying (7):

$$
\pi(g) \sigma(\widehat{g}) \pi(g)^{*}=\langle g, \widehat{g}\rangle \sigma(\widehat{g}) .
$$

One can ask what happens if $\widehat{G}$ is replaced by some other abelian group $H$ with a non-degenerate (but not perfect) pairing

$$
\langle,\rangle: G \times H \rightarrow \mathbb{T},
$$

$\mathbb{T}$ the circle group. This pairing can also be characterized by a continuous homomorphism $H \rightarrow \widehat{G}$; non-degeneracy of the pairing means this map is injective. The simplest example is $G=H=\mathbb{Z}$ with a dense embedding $H \hookrightarrow \mathbb{T}$ sending the generator to $e^{2 \pi i \lambda}, \lambda \notin \mathbb{Q}$. The associated $C^{*}$-algebra is called an irrational rotation algebra or noncommutative torus. (The term noncommutative torus also encompasses the more general situation of the twisted group $C^{*}$-algebra $C^{*}\left(\mathbb{Z}^{n}, \omega\right)$, with $\omega$ a 2-cocycle (which we can take [30] to be given by a skew-symmetric bilinear form) on $\mathbb{Z}^{n}$ with values in $\mathbb{T}$. The irrational rotation algebras correspond to taking $n=2, \omega$ defined by $\omega(a, b)=e^{2 \pi i \lambda\left(a_{1} b_{2}-a_{2} b_{1}\right)}$.)

In the situation of a dense but non-closed embedding $H \hookrightarrow \widehat{G}$, the Morita equivalence with $\mathbb{C}$ breaks down, but a vestige of it remains. A precise statement is as follows:

TheOREM 6.1. Let $H$ and $G$ be locally compact abelian groups, and suppose $\psi: H \hookrightarrow \widehat{G}$ is a continuous embedding with dense but non-closed range. Then the universal $C^{*}$-algebra $C^{*}(G, H, \psi)$ generated by covariant pairs of unitary representations $\pi$ of $G$ and $\sigma$ of $H$ satisfying the commutation relation

$$
\pi(g) \sigma(h) \pi(g)^{*}=\psi(h)(g) \sigma(h)
$$

is simple and has a faithful lower-semi-continuous semifinite trace $\tau$, and $\tau$ is unique up to scalar multiples.

The history of this theorem is a bit complicated. Many cases are included in $[\mathbf{1 7}],[\mathbf{1 0}]$, and $[\mathbf{6 8}]$, all of which take a very different point of view from the one in this paper. The very important special case where $G$ and $H$ are compactly generated abelian Lie groups (products of finitely generated discrete abelian groups, tori, and vector groups) was treated in detail by Pukánszky [46], who used this as a basis for his entire theory of non-type I representations of solvable (or even completely general) connected Lie groups. Baggett and Kleppner [1] obtained the general simplicity criterion for twisted group $C^{*}$-algebras of abelian groups, of which this is a special case, and Kleppner [31] stated the unique trace property, but his proof was incomplete. A complete proof of this theorem was finally given by Green [15], who also showed how to use this result as a building block in a structure theory for $C^{*}$-algebras of connected Lie groups.

The upshot of this theorem is that while $C^{*}(G, H, \psi)$ has many non-equivalent irreducible representations, it has a unique quasi-equivalence class of traceable factor representations (of type II), which should be viewed as the good replacements for the Schrödinger representation in the Stone-von Neumann Theorem. 


\section{Supersymmetry and analogues of Stone-von Neumann}

We conclude by mentioning some other analogues or generalizations of the Stone-von Neumann Theorem. The Heisenberg commutation relations (or canonical commutation relations, usually abbreviated CCR) are appropriate for free bosons. One can equally well consider the canonical anticommutation relations (CAR), appropriate for free fermions, or a more realistic mixture of both bosons and fermions. This is a necessary prerequisite for studying what physicists call supersymmetry, which is a kind of symmetry allowing mixing of the two kinds of particles. We will consider first the analogues of the Stone-von Neumann Theorem for fermionic systems with finitely many degrees of freedom, then the CAR and CCR with infinitely many degrees of freedom, needed for quantum statistical mechanics and quantum field theory, and then finally mixed systems of both bosons and fermions, and supersymmetry.

7.1. Fermionic systems with finitely many degrees of freedom. The canonical anticommutation relations (or CAR) are given by

$$
a_{j} a_{k}+a_{k} a_{j}=0, \quad a_{j} a_{k}^{*}+a_{k}^{*} a_{j}=\delta_{j k}, \quad j, k \leq m,
$$

where $m$ is the "number of degrees of freedom," which we allow to be a positive integer or $\aleph_{0}$. These relations arise from the study of systems of $m$ fermions, particles (like electrons) obeying the Pauli exclusion principle, and were first formulated by Jordan and Wigner [28]. They arise, for example, if the $a_{j}$ 's are the "creation operators" or "annihilation operators" of a fermion field (in quantum field theory). The operators $a_{j}$ are supposed to be closed, densely defined operators on a Hilbert space $\mathcal{H}$; since $(13)$ implies that $a_{j} a_{j}^{*}+a_{j}^{*} a_{j}=1$, they are bounded with norm $\leq 1$, and in fact $a_{j}+a_{j}^{*}$ and $i\left(a_{j}-a_{j}^{*}\right)$ are each self-adjoint with square 1 and anticommute. Thus the $a_{j}+a_{j}^{*}$ and $i\left(a_{j}-a_{j}^{*}\right), j \leq m$ generate (if $m<\infty$ ) a Clifford algebra over $\mathbb{C}$ on $2 m$ generators, isomorphic (as a $C^{*}$-algebra) to the matrix algebra $M_{2^{m}}(\mathbb{C})$, or to $\bigotimes_{j=1}^{m} M_{2}(\mathbb{C})$. We can call this the $C A R$ algebra with $m$ degrees of freedom. So again one has a Morita equivalence with $\mathbb{C}$, i.e., the analogue of Stone-von Neumann holds for fermions.

One can formulate things in a slightly more canonical way by getting rid of the implicit choice of basis in (13). Let $V$ be a finite-dimensional complex Hilbert space of dimension $m$, and for $f \in V$, suppose one has an operator $a(f)$, with $f \mapsto a(f)$ linear, satisfying the relations

$$
a(f) a(g)+a(g) a(f)=0, \quad a(f) a(g)^{*}+a(g)^{*} a(f)=\langle f, g\rangle, \quad f \in V .
$$

These are exactly the relations one gets from (13) upon defining $a\left(\sum \lambda_{j} e_{j}\right)=$ $\sum \lambda_{j} a_{j}$, for $\left\{e_{j}\right\}$ an orthonormal basis of $V$. Then $a(f)-a(f)^{*}$ is skew-adjoint, and

$$
\left\{a(f)-a(f)^{*}, a(g)-a(g)^{*}\right\}=-\langle f, g\rangle-\langle g, f\rangle=-2 \operatorname{Re}\langle f, g\rangle,
$$

where $\{a, b\}=a b+b a$ denotes the anticommutator. Thus if $V_{\mathbb{R}}$ denotes the "realification" of $V$, a real Hilbert space of dimension $2 m$ which as a vector space over $\mathbb{R}$ coincides with $V$ and has real inner product $(f, g)=\operatorname{Re}\langle f, g\rangle$, we see that the $b(f)=a(f)-a(f)^{*}$ satisfy $\{b(f), b(g)\}=-2(f, g)$, the relations for the real Clifford algebra $\operatorname{Cliff}\left(V_{\mathbb{R}}\right)$ (with the generators $b(f)$ skew-adjoint). Furthermore, the real orthogonal group $O\left(V_{\mathbb{R}}\right)$ acts on $V_{\mathbb{R}}$, preserving all these relations. The 
complex structure on $V$ induces a canonical orientation on $V_{\mathbb{R}}$, so the special orthogonal group $S O\left(V_{\mathbb{R}}\right)$ thus plays the same role for fermions that the symplectic group $\operatorname{Sp}(2 m, \mathbb{R})$ plays for a system of $m$ free bosons. We can now formulate the fermionic analogue of the Stone-von Neumann and Shale-Weil theorems as follows:

THEOREM 7.1. Let $V$ be a complex Hilbert space of finite dimension $m$, and consider representations on complex Hilbert spaces $\mathcal{H}$ of the canonical anticommutation relations (14) based on $V$ (with $f \mapsto a(f)$ linear) as above. Then all such representations are multiples of a unique irreducible representation on a Hilbert space $\mathcal{H}_{0}$ of dimension $2^{m}$, and the projective representation of $S O\left(V_{\mathbb{R}}\right)$ on $\mathcal{H}_{0}$ defined by (9) lifts to a true representation of the double cover $\operatorname{Spin}\left(V_{\mathbb{R}}\right)$ (the spin representation).

I am not sure how early the close analogy with the Stone-von Neumann Theorem was appreciated, but certainly it is made explicit in $[\mathbf{1 3}]$ and in [53]. The analogy is discussed in detail in [25].

7.2. The CAR and CCR with infinitely many degrees of freedom. In the case of infinitely many degrees of freedom, Stone-von Neumann no longer holds for either bosons or fermions, but an analogue of the Baggett-Kleppner theorem still holds; one sometimes gets a simple $C^{*}$-algebra with unique trace.

For systems with infinitely many degrees of freedom, the theory of representations of the CAR is somewhat easier than that for the CCR. Investigation of this case goes back to [12] and [54]. Shale and Stinespring consider representations of the Clifford algebra $\operatorname{Cliff}\left(V_{\mathbb{R}}\right)$ as above, but now with $V$ a separable infinitedimensional Hilbert space. In particular, they characterize the states of this algebra which are invariant under the unitary group $U(V)$. These form a 1-parameter family $E_{t},-1 \leq t \leq 1, t=0$ corresponding to the tracial state (see below), $t=1$ corresponding to the "vacuum state," and $t=-1$ corresponding to the "antivacuum state." The vacuum state is described already in [12]; it corresponds (as the name suggests) to the "zero particle" vector in the Fock representation, which in turn was defined rigorously by Cook [7], following the ideas of Fock [11]. (The vacuum vector is characterized up to scalar multiples by the properties that it is annihilated by all the annihilation operators, and that iterated products of the creation operators acting on it generate the whole space.) The anti-vacuum state is similar, but corresponds to interchanging the creation operators with their adjoints, the annihilation operators.

A more convenient way to deal with the CAR uses UHF algebras, introduced by Glimm in [14]. As we have seen, the CAR with $m$ degrees of freedom generate a finite-dimensional $C^{*}$-algebra isomorphic to $M_{2^{m}}(\mathbb{C}) \cong \bigotimes_{j=1}^{m} M_{2}(\mathbb{C})$. If we consider the effect of increasing the number of degrees of freedom by one, this corresponds to a unital mapping $M_{2^{m}}(\mathbb{C}) \hookrightarrow M_{2^{m+1}}(\mathbb{C}) \cong M_{2}\left(M_{2^{m}}(\mathbb{C})\right)$ which is equivalent to $a \mapsto\left(\begin{array}{ll}a & 0 \\ 0 & a\end{array}\right)$. On passage to the limit, we get an infinite-dimensional unital $C^{*}$-inductive limit $A=\lim M_{2^{m}}(\mathbb{C})$ of matrix algebras, or a UHF algebra (this stands for "uniformly hyperfinite"), as studied in [14]. This particular algebra is called the $2^{\infty}$ UHF algebra or the fermion UHF algebra, sometimes just the $C A R$ algebra, and can be written heuristically as $\bigotimes_{j=1}^{\infty} M_{2}(\mathbb{C})$. Guichardet [18] put this "infinite tensor product" point of view on a rigorous basis, and made the connection between the work of Shale-Stinespring and that of Glimm. The algebra 
$A$ is simple, nuclear, and has a unique (finite) trace, which is the Shale-Stinespring state $E_{0}$. One possible substitute for the Stone-von Neumann Theorem for systems of infinitely many fermions is thus that $A$ has a unique quasi-equivalence class of traceable factor representations. The algebra $A$ does have a huge number of unitarily inequivalent irreducible representations, which can be viewed as corresponding to physically inequivalent systems. However, Powers proved that there is a sense in which all of these are equivalent to one another, and one can view this as a deeper analogue of the Stone-von Neumann Theorem:

Theorem 7.2 ([44, Corollary 3.8, p. 157]). Let $A$ be a UHF algebra, or in particular the fermion UHF algebra. Then the *-automorphism group of $A$ acts transitively on the set of pure states of $A$.

The theory of representations of the CCR with infinitely many degrees of freedom, which more directly corresponds to the situation of the Stone-von Neumann Theorem, is substantially more complicated, largely because there is no obvious choice of a $C^{*}$-algebra whose representations correspond to those of the relations. We may explain the issues involved as follows. The Stone-von Neumann Theorem comes from quantizing a finite-dimensional symplectic vector space. Thus to study the CCR with infinitely many degrees of freedom, we may consider a separable infinite-dimensional complex Hilbert space $V$ with inner product $\langle$,$\rangle . As above,$ the realification $V_{\mathbb{R}}$ of $V$ is a real Hilbert space, but it also carries a non-degenerate alternating bilinear form $B(f, g)=\operatorname{Im}\langle f, g\rangle$. A representation of the CCR based on $V$ (again we use the "Weyl integrated form") is given by a map $W$ from $V$ (or at least a dense subspace) to the unitaries on a complex Hilbert space $\mathcal{H}$ satisfying the analogue of (5) and (8):

$$
W\left(z_{1}\right) W\left(z_{2}\right)=e^{i B\left(z_{1}, z_{2}\right) / 2} W\left(z_{1}+z_{2}\right),
$$

with $B$ as above the imaginary part of the complex inner product. One also needs to impose some continuity or measurability conditions on the map $W$, at least that its restriction to each finite-dimensional subspace should be strongly continuous. In other words, we want a projective representation of $V_{\mathbb{R}}$ (with respect to a suitable topology) with cocycle defined by $e^{i B / 2}$.

Initial studies of the representations of the CCR were undertaken in $[\mathbf{1 3}]$, [7], [51], and [53]. These authors did for the CCR pretty much what had been done for the CAR. For instance, Cook [7], again following Fock [11], defined the Fock representation, and all these authors showed that there are a large number of other inequivalent irreducible representations. A particularly interesting result is a uniqueness theorem of Segal:

Theorem 7.3 ([52, Theorem 3]). Suppose $W$ is a continuous representation on a Hilbert space $\mathcal{H}$ of the CCR (16) based on $V$. Also assume that there is a continuous one-parameter unitary group $U(t), t \in \mathbb{R}$ on $V$, whose self-adjoint generator is non-negative, with no point spectrum at 0 . Suppose there is a continuous one-parameter unitary group $\Gamma(t), t \in \mathbb{R}$ on $\mathcal{H}$, whose self-adjoint generator is non-negative ${ }^{23}$ and satisfies

$$
\Gamma(t) W(z) \Gamma(t)^{*}=W(U(t) z), \quad z \in V, t \in \mathbb{R} .
$$

${ }^{23}$ In practice this is supposed to be the Hamiltonian. The one-parameter unitary group $\Gamma(t)$ gives the time evolution of the system. 
Finally, suppose there is a unit vector $\xi \in \mathcal{H}$ which is cyclic for $W$ and invariant under all $\Gamma(t)$. Then $W$ is unitarily equivalent to the Fock representation.

Attempts to systematize the work on representations of the CCR by analyzing the structure of various related $C^{*}$-algebras may be found in $[\mathbf{2 9}],[\mathbf{4 0}],[\mathbf{5 5}]$. Unfortunately $V_{\mathbb{R}}$ is not locally compact, so the usual techniques of group representation theory do not all apply here. As Kastler points out carefully in [29], there is no single "CCR algebra" with all of the properties one would want (separability, covariance with respect to $U(V)$, etc.). Perhaps the best of the alternatives proposed there is the algebra which Kastler (in our notation) calls $\mathcal{K}\left(V_{\mathbb{R}}, B\right)$. This is the universal $C^{*}$-algebra completion of the subalgebra of the measure algebra of $V_{\mathbb{R}}$ generated by all absolutely continuous measures on finite-dimensional subspaces. The multiplication here is given by twisted convolution of measures, the twist coming from the 2-cocycle $e^{i B / 2}$.

Here I would like to suggest a new approach to representation theory of the CCR, motivated by the work of Strătilă and Voiculescu on representations of infinite-dimensional unitary groups [58]. This sacrifices something in terms of canonicity (compared to the approaches of Kastler, Manuceau, and Slawny), but it has the advantage of providing a very concrete algebra whose representation theory we can analyze in detail using the theory in $[\mathbf{2}]$. Namely, consider representations $W$ of the CCR modeled on $V_{\mathbb{R}}$ (as in (16)), where $V$ is an infinite-dimensional separable Hilbert space $V$, and fix an orthonormal basis $\left\{e_{k}\right\}$ for $V$. For each positive integer $n$, let $V_{n}$ be the $\mathbb{C}$-span of $e_{1}, \cdots, e_{n}$, and let $X_{n}$ be the $\mathbb{R}$-span of $e_{1}, \cdots, e_{n}$. Then $V_{1} \subset V_{2} \subset \cdots$ and $V_{n} \nearrow V$. By the Stone-von Neumann Theorem (and its proof), $\left.W\right|_{V_{n}}$, integrated against Schwartz functions on $V_{n}$, generates a $C^{*}$-algebra isomorphic to $\mathcal{K}\left(L^{2}\left(X_{n}\right)\right)$, the compact operators on the space of the Schrödinger representation. Thus if we follow the idea of Kastler and consider the image of the measure algebra of $V$ generated by absolutely continuous measures on finite-dimensional subspaces, but take these finite-dimensional subspaces to be non-zero and only from the flag $V_{1} \subset V_{2} \subset \cdots$, we obtain a $C^{*}$-algebra which is the $C^{*}$-inductive limit of the algebras $A_{n}$, where

$$
A_{n}=\mathcal{K}\left(L^{2}\left(X_{n}\right)\right)+\mathcal{K}\left(L^{2}\left(X_{n-1}\right)\right) \otimes 1_{L^{2}(\mathbb{R})}+\cdots+\mathcal{K}\left(L^{2}\left(X_{1}\right)\right) \otimes 1_{L^{2}\left(\mathbb{R}^{n-1}\right)},
$$

and

$$
\mathcal{B}\left(L^{2}\left(\mathbb{R}^{n}\right)\right) \supset A_{n} \hookrightarrow A_{n+1} \subset \mathcal{B}\left(L^{2}\left(\mathbb{R}^{n+1}\right)\right)
$$

via $a \mapsto a \otimes 1$. Furthermore, $A_{n+1} \supset \mathcal{K}\left(L^{2}\left(X_{n+1}\right)\right)$ and the image of $A_{n}$ splits the quotient map $A_{n+1} \rightarrow A_{n+1} / \mathcal{K}\left(L^{2}\left(X_{n+1}\right)\right)$. We see, therefore, that $A_{n}$ is (for each $n$ ) a type I $C^{*}$-algebra with finite spectrum; in fact, $\widehat{A}_{n}$ is a space with $n$ linearly ordered points, with the $T_{0}$ topology determined by this order. Let $A=\lim A_{n}$, the inductive limit being computed with respect to the maps $A_{n} \rightarrow$

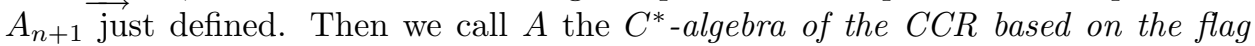
$\left\{V_{n}\right\}$. Note that (unlike the CCR algebras considered in [29], [55], and [40]) $A$ is separable. We will completely determine its structure. In the notation of $[\mathbf{2}], A_{n}$ is a lexicographic direct sum of elementary $C^{*}$-algebras indexed by the ordered set $\{-n+1, \cdots, 0\}$ (with its usual ordering). Thus $A$ is the lexicographic direct sum of elementary $C^{*}$-algebras indexed by the ordered set $-\mathbb{N}$ (the non-positive integers). Thus application of the results of [2] leads to the following classification theorem:

Theorem 7.4. Let $A$ be the $C^{*}$-algebra of the CCR based on an infinite flag, as above. Then $A$ is an $A F$ (approximately finite-dimensional) algebra, and the 
ordered group $K_{0}(A)$ is isomorphic to the lexicographic direct sum $\mathbb{Z}^{(-\mathbb{N})}$ lex . The algebra $A$ is primitive and is not postliminary. In terms of the classification theorem in [2, Theorem 4.13], A is characterized by its "defector" $d \equiv+\infty$.

Every representation of the CCR based on $V$ gives rise to a representation of the $C^{*}$-algebra $A$ of the same type, and conversely, every factor representation of A not factoring though a postliminary quotient gives rise to a representation of the $C C R$ based on $\lim _{n} V_{n} \subset V$. (It will extend to a representation of the CCR based on $V$ itself if a suitable continuity requirement is satisfied.)

Proof. Since each $A_{n}$ is a lexicographic direct sum of elementary $C^{*}$-algebras and the inclusion $A_{n} \hookrightarrow A_{n+1}$ is compatible with the inclusion of ordered sets $\{-n+1, \cdots, 0\} \hookrightarrow\{-n,-n+1, \cdots, 0\}$, it follows by passage to the limit that $A$ is a lexicographic direct sum of elementary $C^{*}$-algebras indexed by the ordered set $-\mathbb{N}$. By $[\mathbf{2}$, Theorem 2.8], $A$ is then AF. Since $-\mathbb{N}$ does not have the descending chain chain condition, it follows by $[\mathbf{2}$, Theorem 2.8] that $A$ is not postliminary, and it follows from the description of the ideals of $A$ in [2, Theorem 2.2] that $A$ is prime (or equivalently, primitive). The calculation of the defector is obvious: $d \equiv+\infty$ since each elementary subquotient of $A$ has dimension $\aleph_{0}$. (See the remark in $[\mathbf{2}$, top of page 264].)

The final statements about the relationship between representations of $A$ and representations of the CCR follows from the way $A$ was constructed and from density of $\lim _{\longrightarrow} V_{n}$ in $V$.

Note, as in the work of Strătilă and D. Voiculescu in [58], that $A$ also carries some "uninteresting" exceptional representations, those factoring through some quotient isomorphic to $A_{n}$ for some $n$. But the very concrete nature of the algebra $A$ makes it possible to recover many results on concrete representations of the CCR more simply. For example, from the fact that $A$ is not postliminary, we immediately deduce that the family of representations of the CCR based on $\lim V_{n}$ is not countably separated, which strengthens the results of Gårding and Wightman [13] on existence of many inequivalent irreducible representations.

7.3. Supersymmetry and the Stone-von Neumann Theorem. The observable universe includes both bosons and fermions, so it has been a goal of physicists, ever since the appearance of $[\mathbf{2 8}]$, to find a way to treat mixed systems in a way that puts both kinds of particles on an equal footing. This makes it possible to contemplate supersymmetries, symmetries that mix the two kinds of particles. Supersymmetry puts constraints on the kinds of field theories that can be contemplated, and has thus served as a useful filter in narrowing the search for a unification of gravity and quantum mechanics.

A basic tenet of supersymmetric theories is that algebras of observables should be $\mathbb{Z} / 2$-graded, with "even" elements corresponding to bosons and "odd" elements corresponding to fermions. Supersymmetries are encoded in actions of "super Lie algebras" and "super Lie groups." There is some dispute in the physics literature about the best way to codify these, but a reasonable formalism was given and developed by Kostant in [32]. For present purposes, a "super Lie algebra" will mean a $\mathbb{Z} / 2$-graded Lie algebra $\mathfrak{g}=\mathfrak{g}_{0}+\mathfrak{g}_{1}$ (over $\mathbb{R}$ or $\mathbb{C}$ ), where $\mathfrak{g}_{0}$ is an ordinary Lie algebra, $\mathfrak{g}_{1}$ is a $\mathfrak{g}_{0}$-module, and there is a symmetric bilinear bracket $[]:, \mathfrak{g}_{1} \times$ $\mathfrak{g}_{1} \rightarrow \mathfrak{g}_{0}$. The graded Jacobi identity has to be satisfied. (For a simple example, start with a $\mathbb{Z} / 2$-graded associative algebra $\mathfrak{g}$ and take the bracket to be the usual 
commutator on $\mathfrak{g}_{0} \times \mathfrak{g}_{0}, \mathfrak{g}_{0} \times \mathfrak{g}_{1}$, and $\mathfrak{g}_{1} \times \mathfrak{g}_{0}$, but the anticommutator on $\mathfrak{g}_{1} \times \mathfrak{g}_{1}$.) A "super Lie group" will be given by a pair $(G, \mathfrak{g})$, where $\mathfrak{g}$ is a super Lie algebra and $G$ is a Lie group with Lie algebra $\mathfrak{g}_{0}$, so that $\mathfrak{g}_{1}$ has a $G$-module structure compatible with its $\mathfrak{g}_{0}$-module structure. (This is not Kostant's definition but is basically equivalent to it, at least when $G$ is connected-see [32, Corollary to Theorem 3.7]. There is no reasonable way to "exponentiate" the odd part of $\mathfrak{g}$.) For example (see [32, Remark 5.3.4] or [35]), there is a "super" version $(H, \mathfrak{h})$ of the Heisenberg group (for $m$ bosonic and $n$ fermionic degrees of freedom) with $H$ the usual Heisenberg group of dimension $2 m+1, \mathfrak{h}_{0}$ its Lie algebra, and $\mathfrak{h}_{1}$ a real vector space of dimension $2 n$ with a (possibly indefinite) symmetric inner product $B{ }^{24}$ The bracket $\mathfrak{h}_{1} \times \mathfrak{h}_{1} \rightarrow \mathfrak{h}_{0}$ takes its values in the center $\mathfrak{z}$ of $\mathfrak{h}_{0}$ and is given by $[x, y]=B(x, y) z$, where $z$ is a basis element for $\mathfrak{z}$.

Now we are ready to state and prove supersymmetric versions of the Stone-von Neumann and Shale-Weil Theorems. These results seem to have been part of the folk literature for some time, so I have not been able to find definitive attributions for them. They may be found essentially in the version stated here in $[\mathbf{3 5}$, pp. 2744-2745], as well as in [19].

ThEOREM 7.5. For any non-negative integers $m$ and $n$, there is a unique unitary equivalence class of irreducible unitary representations $\mu$ of the super-Heisenberg group $(H, \mathfrak{h})$ (for $m$ bosonic and $n$ fermionic degrees of freedom), assuming we have fixed the central character of the representation on $\mathfrak{z}, z \mapsto i \hbar$. Here "unitary" means that $\mu$ is a continuous unitary representation of $H$ and is a *-representation of the CAR, (13), for the fermionic generators.

Proof. By the Stone-von Neumann Theorem and its fermionic analogue (Theorem 7.1), there is one and (up to unitary equivalence) only one unitary representation $\mu$ of $H$ with the correct central character, namely the (outer) tensor product of the Schrödinger representation of $H$ with the unique irreducible representation of Cliff $\left(\mathfrak{h}_{1}\right){ }^{24}$

Now acting by automorphisms on the above super-Heisenberg group is a classical (simple) super Lie group $(\operatorname{Sp}(2 m, \mathbb{R}) \times \operatorname{Spin}(2 n), \mathfrak{o s p}(2 m \mid 2 n))$ ([56, p. 147], [25, Theorems 4 and 5]). The following result can be called the "super Shale-Weil Theorem." Related results may be found in [16] and in [25].

THEOREM 7.6. Let $\mu$, as in Theorem 7.5, be the unique unitary representation of the super-Heisenberg group $(H, \mathfrak{h})$ (for $m$ bosonic and $n$ fermionic degrees of freedom). Assume $\mu$ acts on a Hilbert space $\mathcal{H}$. Then $\mathcal{H}$ carries a compatible unitary representation of the supersymmetry super Lie group $(\mathrm{Mp}(2 m, \mathbb{R}) \times$ $\operatorname{Spin}(2 n), \mathfrak{o s p}(2 m \mid 2 n))$. Here $\operatorname{Mp}(2 m, \mathbb{R})$, often called the metaplectic group, is the double cover of the symplectic group.

ProOF. If we combine the results of section 4 and subsection 7.1 , we see that (9) gives a unitary representation of $\operatorname{Mp}(2 m, \mathbb{R}) \times \operatorname{Spin}(2 n)$ on $\mathcal{H}$. So we just need a compatible action of the odd part of $\mathfrak{o s p}(2 m \mid 2 n)$ on $\mathcal{H}$. As in [25] and in [35, p. 2741], the odd part of $\mathfrak{o s p}(2 m \mid 2 n)$ is canonically isomorphic to $\left(\mathfrak{h}_{0} / \mathfrak{z}\right) \otimes$ $\mathfrak{h}_{1}$. It therefore acts on $\mathcal{H}$ by the span of products of $d \mu\left(P_{j}\right)$ or $d \mu\left(Q_{j}\right)$ with

\footnotetext{
${ }^{24}$ It isn't necessary to have $\mathfrak{h}_{1}$ even-dimensional to define the super Lie algebra, but that's the case we are interested in for purposes of generalizing the Stone-von Neumann Theorem. Even dimensionality of $\mathfrak{h}_{1}$ means $\operatorname{Cliff}\left(\mathfrak{h}_{1}\right)$ has a unique irreducible (complex) representation.
} 
$d \mu\left(a_{k}\right)$ or $d \mu\left(a_{k}\right)^{*}$. Now (as is well known) the infinitesimal action of $\mathfrak{s p}(2 m)$ in the oscillator representation is by the second symmetric power of the action of $\mathfrak{h}_{0}$, and the infinitesimal action of $\mathfrak{s o}(2 n)$ in the spin representation is by the second exterior power of the action of $\mathfrak{h}_{1}$. (See [35, p. 2743], [25], or [19].) But $S^{2}(\mathfrak{h} / \mathfrak{z})$ is isomorphic to $\mathfrak{o s p}(2 m \mid 2 n)$ as a super Lie algebra [35, p. 2741], so the action of the odd part that we have just defined is compatible with the super oscillator representation of $\mathfrak{s p}(2 m) \times \mathfrak{s o}(2 n)$, and we get an action of all of $\mathfrak{o s p}(2 m \mid 2 n)$.

\section{References}

[1] L. Baggett and A. Kleppner, Multiplier representations of abelian groups, J. Functional Analysis 14 (1973), 299-324.

[2] H. Behncke and G. A. Elliott, Lexicographic direct sums of elementary $C^{*}$-algebras, Canad. J. Math. 39 (1987), no. 2, 257-296.

[3] R. J. Blattner, Positive definite measures, Proc. Amer. Math. Soc. 14 (1963), 423-428.

[4] M. Born and P. Jordan, Zur Quantenmechanik, Z. f. Physik 34 (1926), 858-888. Translated (in abridged form) as [60, paper 13, pp. 277-306].

[5] M. Born, W. Heisenberg, and P. Jordan, Zur Quantenmechanik, II, Z. f. Physik 35 (1926), 557-615. Translated as [60, paper 15, pp. 321-385].

[6] L. G. Brown, P. Green, and M. A. Rieffel, Stable isomorphism and strong Morita equivalence of $C^{*}$-algebras, Pacific J. Math. 71 (1977), no. 2, 349-363.

[7] J. M. Cook, The mathematics of second quantization, Trans. Amer. Math. Soc. 74 (1953), no. $2,222-245$.

[8] P. A. M. Dirac, The fundamental equations of quantum mechanics, Proc. Royal Soc. London, Ser. A 109 (Dec. 1, 1925) no. 752, 642-653. Reprinted as [60, paper 13, pp. 307-320].

[9] P. A. M. Dirac, Quantum mechanics and a preliminary investigation of the hydrogen atom, Proc. Royal Soc. London, Ser. A 110 (Mar. 1, 1926) no. 755, 561-579. Reprinted as [60, paper 17, pp. 417-427].

[10] E. G. Effros and F. Hahn, Locally compact transformation groups and $C^{*}$-algebras, Mem. Amer. Math. Soc., No. 75, Amer. Math. Soc., Providence, R.I., 1967.

[11] V. Fock, Konfigurationsraum und zweite Quantelung, Z. f. Physik 75 (1932), 622-647.

[12] L. Gårding and A. Wightman, Representations of the anticommutation relations, Proc. Nat. Acad. Sci. U.S.A. 40 (1954), 617-621.

[13] L. Gårding and A. Wightman, Representations of the commutation relations, Proc. Nat. Acad. Sci. U.S.A. 40 (1954), 622-626.

[14] J. G. Glimm, On a certain class of operator algebras, Trans. Amer. Math. Soc. 95 (1960), 318-340.

[15] P. Green, The local structure of twisted covariance algebras, Acta Math. 140 (1978), no. 3-4, 191-250.

[16] H. Grosse and L. Pittner, A supersymmetric generalization of von Neumann's theorem, J. Math. Phys. 29 (1988), no. 1, 110-118.

[17] A. Guichardet, Caractères des algèbres de Banach involutives, Ann. Inst. Fourier (Grenoble) 13 (1963), 1-81.

[18] A. Guichardet, Produits tensoriels infinis et représentations des relations d'anticommutation, Ann. Sci. École Norm. Sup. (3) 83 (1966), 1-52.

[19] M. Günaydin and S. J. Hyun, Unitary lowest weight representations of the noncompact supergroup $\operatorname{OSp}(2 n \mid 2 m, \mathbb{R})$, J. Math. Phys. 29 (1988), no. 11, 2367-2376.

[20] W. Heisenberg, Über quantentheoretische Umdeutung kinematischer und mechanischer Beziehungen, Z. f. Physik 33 (1925), 879-893. Translated as [60, paper 12, pp. 261-276].

[21] W. Heisenberg, Die physikalischen Prinzipien der Quantentheorie, Hirzel, Leipzig, 1930.

[22] R. Howe, On the character of Weil's representation, Trans. Amer. Math. Soc. 177 (1973), $287-298$.

[23] R. Howe, $\theta$-series and invariant theory, in Automorphic forms, representations and $L$ functions (Oregon State Univ., Corvallis, Ore., 1977), Proc. Sympos. Pure Math., XXXIII, Part 1, Amer. Math. Soc., Providence, R.I., 1979, pp. 275-285.

[24] R. Howe, On the role of the Heisenberg group in harmonic analysis, Bull. Amer. Math. Soc. (N.S.) 3 (1980), no. 2, 821-843. 
[25] R. Howe, Remarks on classical invariant theory, Trans. Amer. Math. Soc. 313 (1989), no. 2, 539-570; Erratum, ibid. 318 (1990), no. 2, 823.

[26] R. Howe, Transcending classical invariant theory, J. Amer. Math. Soc. 2 (1989), no. 3, 535552.

[27] N. Jacobson, Basic algebra, II, W. H. Freeman and Co., San Francisco, Calif., 1980. Second edition, W. H. Freeman and Company, New York, 1989.

[28] P. Jordan and E. P. Wigner, Über das Paulische Äquivalenzverbot, Z. f. Physik 47 (1928), 631-651. Reprinted in E. P. Wigner, Collected Works, A. Wightman, ed., Part A, Volume 1, Springer-Verlag, Berlin and New York, 1993, pp. 109-129.

[29] D. Kastler, The $C^{*}$-algebras of a free Boson field, I: Discussion of the basic facts, Comm. Math. Phys. 1 (1965), 14-48.

[30] A. Kleppner, Multipliers on abelian groups, Math. Ann. 158 (1965), 11-34.

[31] A. Kleppner, Non-type I multiplier representations of abelian groups, unpublished preprint, 1975.

[32] B. Kostant, Graded manifolds, graded Lie theory, and prequantization, in Differential geometrical methods in mathematical physics (Proc. Sympos., Univ. Bonn, Bonn, 1975), Lecture Notes in Math., Vol. 570, Springer, Berlin, 1977, pp. 177-306.

[33] T. Y. Lam, Lectures on modules and rings, Graduate Texts in Math., no. 189, SpringerVerlag, New York, 1999.

[34] G. Lion and M. Vergne, The Weil representation, Maslov index and theta series, Progress in Mathematics, no. 6, Birkhäuser, Boston, Mass., 1980.

[35] W. T. Lo, Super theta functions and the Weil representations, J. Phys. A 27 (1994), no. 8, 2739-2748.

[36] G. W. Mackey, A theorem of Stone and von Neumann, Duke Math. J. 16 (1949), 313-326.

[37] G. W. Mackey, Imprimitivity for representations of locally compact groups, I, Proc. Nat. Acad. Sci. U.S.A. 35 (1949), 537-545.

[38] G. W. Mackey, Induced representations of locally compact groups, I, Ann. of Math. (2) 55 (1952), 101-139.

[39] G. W. Mackey, Unitary representations of group extensions, I, Acta Math. 99 (1958), 265311.

[40] J. Manuceau, $C^{*}$-algèbre de relations de commutation, Ann. Inst. H. Poincaré Sect. A (N.S.) 8 (1968), 139-161.

[41] C. Møglin, M.-F. Vignéras, and J.-L. Waldspurger, Correspondances de Howe sur un corps p-adique, Lecture Notes in Mathematics, No. 1291, Springer-Verlag, Berlin, 1987.

[42] B. Ørsted, Induced representations and a new proof of the imprimitivity theorem, J. Funct. Anal. 31 (1979), no. 3, 355-359.

[43] W. Pauli, Wissenschaftlicher Briefwechsel mit Bohr, Einstein, Heisenberg u. a. (Scientific correspondence with Bohr, Einstein, Heisenberg a. o.), vol. 1, A. Hermann, K. v. Meyenn, and V. F. Weisskopf, eds., Sources in the History of Mathematics and Physical Sciences, no. 2, Springer, New York and Berlin, 1979.

[44] R. T. Powers, Representations of uniformly hyperfinite algebras and their associated von Neumann rings, Ann. of Math. (2) 86 (1967), 138-171.

[45] D. Prasad, A brief survey on the theta correspondence, in Number theory (Tiruchirapalli, 1996), Contemp. Math., 210, Amer. Math. Soc., Providence, RI, 1998, pp. 171-193.

[46] L. Pukánszky, Unitary representations of solvable Lie groups, Ann. Sci. École Norm. Sup. (4) 4 (1971), 457-608.

[47] R. Ranga Rao, On some explicit formulas in the theory of the Weil representation, Pacific J. Math. 157 (1993), no. 2, 335-371.

[48] M. Rieffel, On the uniqueness of the Heisenberg commutation relations, Duke Math. J. 39 (1972), 745-752.

[49] M. Rieffel, Induced representations of $C^{*}$-algebras, Advances in Math. 13 (1974), 176-257.

[50] E. Schrödinger, Über das Verhältnis der Heisenberg-Born-Jordanschen Quantenmechanik zu der meinen, Ann. der Physik (4) 79 (1926), 734-756. Reprinted in E. Schrödinger, Gesammelte Abhandlungen, vol. 3, Vieweg, Vienna, 1984, pp. 143-165.

[51] I. E. Segal, Foundations of the theory of dynamical systems of infinitely many degrees of freedom, I, Mat.-Fys. Medd. Danske Vid. Selsk. 31 (1959), no. 12, 39 pp.

[52] I. E. Segal, Mathematical characterization of the physical vacuum for a linear Bose-Einstein field (Foundations of the dynamics of infinite systems, III), Illinois J. Math. 6 (1962), 500-523. 
[53] D. Shale, Linear symmetries of free boson fields, Trans. Amer. Math. Soc. 103 (1962), 149167.

[54] D. Shale and W. F. Stinespring, States of the Clifford algebra, Ann. of Math. (2) 80 (1964), 365-381.

[55] J. Slawny, On factor representations and the $C^{*}$-algebra of canonical commutation relations, Comm. Math. Phys. 24 (1972), 151-170.

[56] S. Sternberg, Some recent results on supersymmetry, in Differential geometrical methods in mathematical physics (Proc. Sympos., Univ. Bonn, Bonn, 1975), Lecture Notes in Math., Vol. 570, Springer, Berlin, 1977, pp. 145-176.

[57] M. H. Stone, Linear transformations in Hilbert space, III: operational methods and group theory, Proc. Nat. Acad. Sci. U.S.A. 16 (1930), 172-175.

[58] Ş. Strătilă and D. Voiculescu, Representations of AF-algebras and of the group $U(\infty)$, Lecture Notes in Math., vol. 486, Springer-Verlag, Berlin-New York, 1975.

[59] S. J. Summers, On the Stone-von Neumann uniqueness theorem and its ramifications, in John von Neumann and the Foundations of Quantum Physics, M. Rédei and M. Stöltzner, eds., Vienna Circle Institute Yearbook 8/2000, Kluwer, Dordrecht, 2001, pp. 135-152.

[60] B. L. van der Waerden, ed., Sources of Quantum Mechanics, North-Holland, Amsterdam, 1967. Reprinted by Dover, Classics of Science, vol. 5, 1968.

[61] J. von Neumann, Mathematische Begründung der Quantenmechanik, Nachrichten Göttingen (1927), 1-57. Reprinted in J. von Neumann, Collected Works, vol. 1, A. H. Taub, ed., Pergamon Press, New York and Oxford, 1961, pp. 151-207.

[62] J. von Neumann, Die Eindeutigkeit der Schrödingerschen Operatoren, Math. Ann. 104 (1931), 570-578. Reprinted in J. von Neumann, Collected Works, vol. 2, A. H. Taub, ed., Pergamon Press, New York and Oxford, 1961, pp. 220-229.

[63] J.-L. Waldspurger, Démonstration d'une conjecture de dualité de Howe dans le cas $p$-adique, $p \neq 2$, in Festschrift in honor of I. I. Piatetski-Shapiro on the occasion of his sixtieth birthday, Part I (Ramat Aviv, 1989), Israel Math. Conf. Proc., vol. 2, Weizmann, Jerusalem, 1990, pp. 267-324.

[64] A. Weil, Sur certains groupes d'opérateurs unitaires, Acta Math. 111 (1964), 143-211.

[65] H. Weyl, Gruppentheorie und Quantenmechanik, Hirzel, Leipzig, 1928. Zweite umgearbeitete Auflage, 1931.

[66] H. Wielandt, Über die Unbeschränktheit der Operatoren der Quantenmechanik, Math. Ann. 121 (1949), 21.

[67] A. Wintner, The unboundedness of quantum-mechanical matrices, Physical Rev. (2) 71 (1947), 738-739.

[68] G. Zeller-Meier, Produits croisés d'une $C^{*}$-algèbre par un groupe d'automorphismes, J. Math. Pures Appl. (9) 47 (1968), 101-239.

Department of Mathematics, University of Maryland, College Park, MD 207424015

E-mail address: jmr@math.umd.edu

$U R L$ : http://www.math. umd.edu/ jmr 